\title{
Clonación, expresión, caracterización y modelado de esterasas putativas de Corynebacterium glutamicum ATTC 13032
}

Inmaculada Navarro-González

Departamento de Bioquímica y Biología Molecular-A, Facultad de Biología, Universidad de Murcia, Campus Espinardo, 30100 Murcia, España.

Correspondencia
I. Navarro-González
E-mail: inmaculada.navarro@um.es
Tel: +34 868884765
Fax: +34 868884765

Recibido: 26 julio 2012

Aceptado: 30 noviembre 2012

Publicado on-line: 12 diciembre 2012

\section{Resumen}

El objetivo de este trabajo fue clonar, expresar, purificar y caracterizar algunos genes de Corynebacterium glutamicum ATCC13032, identificados como enzimas de la superfamilia de las esterasas/lipasas. De todos los genes clonados, solo se pudo estudiar la proteína codificada por el gen $\mathrm{Cg} 2528$. Se purificó y caracterizó la proteína. Su máxima actividad la presentó $40^{\circ} \mathrm{C}$ y a pH 8 . Presentó una gran estabilidad en presencia de disolventes orgánicos como el DMSO y el metanol. Con respecto al análisis cinético, presentó una mayor actividad sobre los $p$-nitrofenil ésteres y los naftilos que sobre el S-methiltiobutanoato. Se dilucidó la triada catalítica alineando la secuencia de aminoácidos. La estructura tridimensional se predijo usando la esterasa (2OGS) de Geobacillus stearothermophilus como molde.

Palabras clave: Esterasas, Purificación, Caracterización bioquímica, Clonación.

\begin{abstract}
Cloning, expression, characterization and modeling of putatives esterases from Corynebacterium glutamicum ATCC 13032

The aim of this work was to clone, express, purifiy and characterize some genes from Corynebacterium glutamicum ATCC13032, identified as enzymes of the superfamily of esterases/lipases. Among all cloned genes, only the protein encoded by the gene $\mathrm{Cg}-2528$ could be studied. The protein was purified and characterized. The optimum activity was observed at $40^{\circ} \mathrm{C}$ and $\mathrm{pH}$ 8. The enzyme showed a high activity in presence of organic solvents, such as DMSO or methanol. $P$-nitrophenyl ester and naphthyl ester derivatives were found to be better substrates than S-methyl tiobutanoate. The catalytic triad was predicted by amino acid sequence alignment and the structure modeling was performed using esterase (2OGS) form Geobacillus stearothermophilus as template.
\end{abstract}

Key words: Esterase, Purification, Biochemical characterization, Cloning. 


\section{Introducción}

En 1957, Kinoshita y otros colaboradores, aislaron en Japón una bacteria del aceite, que segregaba grandes cantidades del aminoácido L-glutámico en el medio donde crecía. Por este motivo y por su forma de bastoncillo decidieron llamarla Corynebacterium glutamicum.

C. glutamicum es una bacteria Gram-positiva, aeróbica y mesófila, capaz de crecer en una gran variedad de azúcares y disolventes orgánicos. Cuando crece en óptimas condiciones (azúcares, sales, oxígeno, biotina, ácidos grasos saturados de 16 y 18 carbonos y $\mathrm{pH}$ entre 7 y 8 ) la bacteria metaboliza la glucosa y produce el ácido L-glutámico. Este aminoácido es muy usado en la industria alimentaria como glutamato monosódico que es un potenciador del sabor (Kalinowski et al. 2003).

Esta peculiaridad de utilizar la glucosa como fuente de carbono para producir el L-glutámico despertó la atención de los microbiólogos para estudiarla más profundamente $\mathrm{y}$, durante el estudio exhaustivo de la bacteria, se encontraron cepas mutadas; que, además de sintetizar L-glutámico, tienen la capacidad de sintetizar otros L-aminoácidos como la L-lisina, L-treonina, L-metionina, actualmente utilizados como aditivos para piensos de animales, y la L-isoleucina, L-ornitina, L-tirosina y la L-glutamina, empleados en la industria de química fina (Pühler \& Tauch., 2003; Leuchternberger, 1996).

La capacidad que tiene esta bacteria de sintetizar aminoácidos, ha hecho que sea una de las más utilizadas a nivel industrial. Debido a su importancia, un grupo de investigadores alemanes (Kalinowski et al. 2003) secuenciaron todo el genoma de la cepa silvestre ATCC13032, depositándola en la base de datos Gen Bank (NC_006958). Independientemente a los alemanes, otro grupo de investigación japonés secuenció su genoma de forma simultánea. El artículo no ha sido publicado, pero la secuencia del genoma está depositada en la base de datos Gen Bank con el acceso (NC_003450).

El hecho de que varios grupos se pusieran a secuenciar el genoma de la misma bacteria, da una idea de la importancia de este microorganismo.

Aunque es una bacteria muy utilizada industrialmente (Ruckert et al. 2003; Kalinowski et al. 2003), no hay ninguna enzima lipolítica de $C$. glutamicum que haya sido estudiada, a pesar de su procedencia oleosa. Por este motivo, uno de los objetivos de este trabajo ha sido clonar, caracterizar cinéticamente y construir un modelo en 3D de las posibles estructuras de algunas esterasas putativas de este microrganismo, para su posible aplicación industrial.

\section{Material y métodos}

\section{Reactivos, plásmidos y cepas bacterianas}

C. glutamicum ATCC13032 se compró a la colección española de cultivos tipo (CECT 4157).

Los kits de Biología Molecular empleados se compraron a Quiagen (Hilden, Alemania).

La T4 DNA ligasa y los oligodesoxirribonucleótidos diseñados específicamente para aislar cada gen, provienen de Isogen Life Science (IJsselstein, Holanda). Los vectores de expresión (pET$28 \mathrm{a}^{+}, \mathrm{pET}-26 \mathrm{~b}^{+}$y pET- $40 \mathrm{~b}^{+}$se adquirieron en Novagen (Darmstadt, Alemania). La cepa bacteriana de Escherichia coli usada para la clonación y expresión (Rosetta (DE3)) y las enzimas de restricción se compraron a Invitrogen (Frederick, EEUU) y Novagen (Darmstadt, Alemania).

Los sustratos para medir la actividad de las esterasas (2-nitrofenilmiristato, 4-nitrofenillaureato, 4-nitrofenilcaprilato, 4-nitrofenilbutirato, 4-nitrofenilacetato, $\alpha$-naftil acetato, $\beta$-naftil acetato, $\alpha$ naftil butirato y $\beta$-naftil butirato), sustrato de tioesterasas (S-metiltiobutanoato), inhibidores específicos de aminoácidos (paraoxon, eserina, pepstatina A, PGO (fenilglicosal monohidradato), DEPC (dietil pirocarbonato), PLP (piridoxal 5-fosfato), PMFS (fluoruro de fenilmetilsulfonilo), iones en forma de sales $\left(\mathrm{Mn}^{2+}, \mathrm{Mg}^{2+}, \mathrm{Hg}^{2+}, \mathrm{Cu}^{2+}, \mathrm{Zn}^{2+}, \mathrm{Ca}^{2+}\right.$ y $\mathrm{Na}^{2+}$ ), EDTA, (IPTG) isopropil- $\beta$-D-tiogalactopiranósido, kanamicina, cloranfenicol, marcadores de peso molecular para experimentos de filtración en gel, el inhibidor de proteasas, el ácido bicinconínico, (BSA) albúmina de suero bovino, DTNB (acido 5-5'-bisditio nitrobenzoico) y el bromuro de etidio se compraron a Sigma (Madrid, España).

Las sales y tampones inorgánicos se adquirieron de Fluka (Buchs, Suiza). Los reactivos para electroforesis de proteínas se obtuvieron de BioRad (Hercules, EEUU). El agua usada se purificó a través de un sistema Milli-Q de Millipore (Bedford, EEUU). Los medios de cultivo se adquirie- 
ron a Laboratorios Conda (Madrid, España).

\section{Clonación y expresión de las proteínas}

Las técnicas de clonación y transformación utilizadas fueron descritas por Sambrook et al. (1989).

Para la clonación del gen $\mathrm{Cg} 0120$ se utilizaron los oligonucleótidos o cebadores complementarios a los extremos 5'y 3'del gen.

Cg0120-up (5'CCCCATATGATGTTTCTCACA CTCTCAGGAGCT3') derivado del extremo 5'de la secuencia del gen.

Cg0120-down (5'CGGATCCCTAGACGGCGT CGAGAAATCG3') derivado del extremo 3 'de la secuencia del gen.

En los cebadores se incluyó la secuencia de reconocimiento para las enzimas de restricción NdeI para Cg0120-up y BamHI para Cg0120-down.

Para la clonación del gen Cg1021 se utilizaron los oligonucleótidos o cebadores complementarios a los extremos 5'y 3'del gen.

Cg1021-up (5'CCGAATTCGTCCTCCCATGTC CGCT3') derivado del extremo $5^{\prime}$ de la secuencia del gen.

Cg1021-down (5'TTAGCGAAGGTGCAGGGTGAAAAAAAGCTTGG3') derivado del extremo 3 'de la secuencia del gen.

En los cebadores se incluyó la secuencia de reconocimiento para las enzimas de restricción EcoRI para Cg1021-up y HindIII para cg1021-down.

Para la clonación del gen Cg1294 se utilizaron los oligonucleótidos o cebadores complementarios a los extremos 5'y 3'del gen.

Cg1294-up (5'CCAAGCTTTAATGCCTCAAAT CCCTGCCCAGAATTT3') derivado del extremo 5 'de la secuencia del gen.

Cg1294-down (5'GCTCGAGTTATCTGGTTTG GTTCAGGAAGCTCAT3') derivado del extremo 3 'de la secuencia del gen.

En los cebadores se incluyó la secuencia de reconocimiento para las enzimas de restricción HindIII para Cg1294-up y XhoI para Cg1294down.

Para la clonación del gen Cg2528 se utilizaron los oligonucleótidos o cebadores complementarios a los extremos 5'y 3'del gen.

Cg2528-up (5' CCAAGCTTGTATGTCTGCC ACCTCTTCCGTCACC $3^{\prime}$ ) derivado del extremo 5 'de la secuencia del gen.

Cg2528-down (5' CCCTCGAGCTAAAAAG CCAGGCGCACC ATG3') derivado del extremo 3'de la secuencia del gen.

En los cebadores se incluyó la secuencia de reconocimiento para las enzimas de restricción HindIII para Cg2528-up y XhoI para Cg2528-down.

Los genes amplificados se obtuvieron mediante PCR (Minicycler Research MJ, EEUU) usando como molde el genómico obtenido de C. glutamicum ATCC13032. Los productos de PCR amplificados se digirieron con sus enzimas de restricción especificas en cada caso y purificados mediante su aislamiento en gel de agarosa, e insertados en un vector de expresión (pET-28a $\mathrm{a}^{+}, \mathrm{pET}^{-26 \mathrm{~b}^{+}}$y pET$40 \mathrm{~b}^{+}$), previamente digerido con los mismos enzimas de restricción. La reacción de ligación entre el vector y el inserto se realizó guardando una relación 2:1 (inserto:vector) a $16{ }^{\circ} \mathrm{C}$ durante 16 horas. Estos plásmidos se utilizaron para transformar en células electrocompetentes de E. coli Rosetta, las cuales se sembraron en placas de LB (Luria Broth) agar con $50 \mu \mathrm{g} / \mathrm{mL}$ kanamicina y 34 $\mu \mathrm{g} / \mathrm{mL}$ cloranfenicol y se incubaron 12 horas a 37 ${ }^{\circ} \mathrm{C}$.

Para identificar los clones se realizaron PCR de colonias, y los clones positivos se pusieron a crecer en LB con los dos antibióticos anteriores a $37{ }^{\circ} \mathrm{C}$ hasta alcanzar una densidad óptica de 0,9 . En este momento se añadió el IPTG a $0,5 \mathrm{mM}$, para inducir y expresar la proteína a $30{ }^{\circ} \mathrm{C}$ durante 6 horas a 150 r.p.m.

La preparación del extracto enzimático inicial se hizo poniendo $0,25 \mathrm{~g}$ de masa bacteriana en 25 $\mathrm{ml}$ de tampón fosfato potásico $50 \mathrm{mM} \mathrm{pH} \mathrm{7.} \mathrm{El} \mathrm{tu-}$ bo que contenía esta suspensión se introdujo en hielo y la suspensión se sonicó durante 1 minuto unos 4 ciclos, a la máxima potencia, utilizando un sonicador Cole-Palmer serie 4710. El lisado celular se centrifugó a 11000 g 20 minutos a $4^{\circ} \mathrm{C}$ y se colectó el sobrenadante, para medir actividad esterasa.

Todos los clones obtenidos se almacenaron a $-80{ }^{\circ} \mathrm{C}$ en un ultracongelador (UltraLow MDFU3086, Sanyo Electric, Japón) en forma de glicerol stock (40\% glicerol) y se secuenciaron para verificar su correcta clonación.

\section{Cultivos de alta densidad}

Para producir una mayor cantidad de proteína se realizaron cultivos bacterianos de alta densidad en $1 \mathrm{~L}$ de medio TB (Terrrific Broth) con kanamicina y cloranfenicol en un matraz aforado de $5 \mathrm{~L}$, bajo las condiciones optimas de expresión de cada pro- 
teína.

Una vez que la proteína se expresó, el cultivo bacteriano se centrifugó a 5000 g a $4{ }^{\circ} \mathrm{C}$ durante 20 minutos, para someterlo a la acción de un homogenizador de bolas de vidrio (Mini Zetall, Netzsch) y obtener así el extracto enzimático.

Tras romper las células con un homogenizador con bolas de vidrio, el extracto enzimático se sometió a la acción de DNasa $\left(750 \mathrm{U} \mathrm{mL}^{-1}\right.$ por cada $15 \mathrm{~g}$ células húmedas) para eliminar los ácidos nucleicos. Tras la incubación con DNasa durante 60 minutos a temperatura ambiente en agitación débil, el extracto se centrifugó a $5000 \mathrm{~g}$ a $4{ }^{\circ} \mathrm{C}$ durante 20 minutos.

\section{Determinación y análisis de proteínas}

La cuantificación de proteína se llevó a cabo por el ensayo del ácido bicinconínico (Smith et al. 1985).

La electroforesis bajo condiciones desnaturalizantes se realizó siguiendo el método Laemmli (1970).

Para el cálculo del porcentaje de proteína recombinante expresada y el peso molecular del monómero, se realizó un gel de electroforesis y posteriormente se efectuó en análisis de este en el equipo Image Quant TL (Amersham Biosciences, Uppsala, Suecia).

\section{Purificación y determinación del peso molecular de la proteína}

Las proteínas se purificaron mediante precipitación con sulfato de amonio. Primeramente, se realizaron experimentos para seleccionar el porcentaje de saturación con sulfato de amonio más adecuado, probándose un rango entre $20 \%$ y $90 \%$ de saturación.

El cálculo de los pesos moleculares se realizó por filtración en gel. Para ello se usó una columna Tricorn Superdex 200 10/300 GL (Amersham Biosciences, Uppsala, Suecia) equilibrada con tampón fosfato potásico $50 \mathrm{mM}$ conteniendo $\mathrm{NaCl}$ 0,15 M. Los experimentos se realizaron en un equipo ÄKTA Purifier (Amersham Biosciences, Uppsala, Suecia) a una velocidad de flujo de $0,5 \mathrm{~mL} / \mathrm{min}$. Los cálculos se hicieron comparando con la recta de calibración elaborada usando un Kit de marcadores de peso molecular (Sigma, St. Louis, EEUU). Los marcadores utilizados fueron aldolasa, $158 \mathrm{kDa}$; albúmina de suero bovina, 66 $\mathrm{kDa}$; anhidrasa carbónica $29 \mathrm{kDa}$; citocromo C,
12 kDa. Para calcular el volumen muerto de la columna se utilizó el azul dextrano.

\section{Medidas de actividad enzimática}

La actividad enzimática se midió por la liberación de $p$-nitrofenol a $405 \mathrm{~nm}$ con sustratos cromogéni$\cos$ artificiales (4-nitrofenilmiristato, 4-nitrofenillaureato, 4-nitrofenilcaprilato, 4-nitrofenilbutirato, 4-nitrofenilacetato). La reacción estándar ( $1 \mathrm{~mL}$ ) contenía $20 \mu \mathrm{l}$ de sustrato (stock $5 \mathrm{mM}$ ), $100 \mu 1$ de extracto enzimático y $880 \mu 1$ de tampón fosfato potásico $100 \mathrm{mM}$ a $\mathrm{pH}$ 7. A este $\mathrm{pH}$, el épsilon del $p$-nitrofenol es de $12000 \mathrm{M}^{-1} \mathrm{~cm}^{-1}$.

Al mismo $\mathrm{pH}$, también se usaron otros sustratos como los naftilos ( $\beta$-naftil acetato, $\alpha$-naftil acetato, $\beta$-naftil butirato y el $\alpha$-naftil butirato) siguiendo el método descrito por Martínez-Martínez et al. (2008). Se define una unidad de esterasa como la cantidad de enzima que es capaz de liberar un $\mu \mathrm{mol}$ por minuto de $p$-nitrofenol a $25^{\circ} \mathrm{C}$, bajo las condiciones de ensayo especificadas.

La actividad tioesterasa se midió empleando el S-metiltiobutanoato como sustrato y DTNB, según Lozada-Ramírez et al. 2006.

\section{Características cinéticas}

El pH óptimo de actuación de la enzima y la especificidad de sustrato se midieron a $348 \mathrm{~nm}$, con los $p$-nitrofenoles, a $30^{\circ} \mathrm{C}$, con un coeficiente molar de $5300 \mathrm{M}^{-1} \mathrm{~cm}^{-1}$. (Mandrich et al. 2005). Los tampones usados fueron de $100 \mathrm{mM}$, con un $\mathrm{pH}$ comprendido entre 3 y 5,5 para el tampón citrato, entre 6 y 7,5 para el fosfato potásico, $\mathrm{pH} 8$ para el Tris-clorhídrico y de 8,5 a 11 para el glicina$\mathrm{NaOH}$.

El efecto de la temperatura sobre la actividad catalítica de la enzima se realizó en un espectrofotómetro UV/ Vis Uvikon con baño de agua recirculante para atemperar el medio de reacción a cada temperatura de medida antes de iniciar la reacción enzimática. El rango de temperaturas ensayadas fue de $20^{\circ} \mathrm{C}$ hasta $60^{\circ} \mathrm{C}$.

La termoestabilidad enzimática se estudió colocando la enzima a diferentes temperaturas $\left(55^{\circ} \mathrm{C}, 50{ }^{\circ} \mathrm{C}, 45^{\circ} \mathrm{C}, 40^{\circ} \mathrm{C}\right.$ y $\left.42^{\circ} \mathrm{C}\right)$ y sacando alícuotas cada cinco minutos para medir la actividad.

La estabilidad de la enzima frente a diversos compuestos, como detergentes (SDS, Tween 20 y Triton X-100) y solventes orgánicos (metanol, etanol, acetona, 2-propanol, DMSO y acetonitrilo), se investigó midiendo la actividad residual de la 
enzima tras ser incubada a $30{ }^{\circ} \mathrm{C}$, con distintas concentraciones de cada una de las anteriores sustancia (detergentes al $5 \%$ y $10 \%$, los disolventes orgánicos al $10 \%, 20 \%$ y al $30 \%$ ).

El efecto de los cationes sobre la actividad cinética de la enzima se verificó incubando la enzima con los iones en forma de sales a $5 \mathrm{mM}(\mathrm{NaCl}$, $\mathrm{CaCl}_{2}, \mathrm{MgCl}_{2}, \mathrm{AlCl}_{3}, \mathrm{LiCl}, \mathrm{NiCl}, \mathrm{MnCl}_{2}$ y $\mathrm{KCl}$, $\mathrm{HgCl}_{2}$ ), media hora a $30{ }^{\circ} \mathrm{C}$ y midiendo actividad pasado ese tiempo. Paralelamente, se preparó otra batería de muestras para incubarlas en idénticas condiciones que las anteriores, pero transcurridos los 30 minutos se añadió EDTA a $10 \mathrm{mM}$ y se dejó actuar 30 minutos antes de medir la actividad residual. Simultáneamente, se prepararon reacciones de control sin sales ni agentes quelantes para comprobar que la pérdida o ganancia de actividad no dependía de la exposición de la enzima a $30{ }^{\circ} \mathrm{C}$.

Los agentes inhibidores reactivos de aminoácidos específicos usados para conocer que aminoácidos son esenciales para la catálisis fueron el piridoxal 5'-fosfato (PLP) para lisina, fluoruro de fenilmetilsulfonilo (PMFS) para serina, dietil pirocarbonato (DEPC) para histidina, fenilglicoxal (PGO) para arginina y pepstatina-A para el ácido aspártico. La enzima se incubó a dos concentraciones distintas, $0,5 \mathrm{mM}$ y $5 \mathrm{mM}$, a $30^{\circ} \mathrm{C}$ y durante 30 minutos. Transcurridos los 30 minutos, se midió la actividad residual. El paraoxon y la eserina se utilizaron de igual forma, pero con la finalidad de ayudarnos a clasificar las esterasas.

\section{Herramientas bioinformaticas}

Las secuencias de nucleótidos de los genes se obtuvieron de la base de datos del Nacional Center for Biotechnology Information (NCBI: http:// www.ncbi.nlm.nih.gov/). El análisis de homología de las secuencias se realizó usando el programa Clustal W (http://npsa-pbil.ibcp.fr) y, puntualmente, la base de datos Protein Data Bank (http:// www.rcsb.org/pdb). Los alineamientos múltiples se realizaron con el programa ESPript (http://espript.ibcp.fr/ESPript). La secuencia de aminoácidos de las distintas enzimas se enviaron al Expert Protein Análisis System Proteomic Server (http:// www.expasy.ch/swissmod/SWISS-MODEL.html) para la construcción de los modelos tridimensionales. Las figuras se realizaron usando los programas DSViewer (Accelerys, Cambridge, Inglaterra) y Swiss-pdb Viewer (GlaxoSmithkline y
Swiss Institute of Bioinformatics, Basel, Suiza). Los diagramas topológicos se construyeron empleando el programa TOPS (http://www.tops.leeds.ac.uk/).

\section{Resultados y discusión}

\section{Búsqueda de genes de putativas esterasas}

Las secuencias de nucleótidos del genoma de $C$. glutamicum 13032 se obtuvo de la base de datos del Nacional Center For Biotechnology Information (NCBI: http://www.ncbi.nlm.nih.gov/), bajo el locus NC_006958.

Del análisis del genómico, depositado por Kalinowski et al. 2003, se deduce que existen 14 esterasas putativas y de una búsqueda bibliográfica exhaustiva se concluye que ninguna ha sido clonada. Todas las enzimas lipolíticas encontradas pueden verse en la tabla 1 .

\section{Clonación, expresión y purificación}

Una vez estudiados y analizados los genes y las posibles esterasas que codificarían, se clonaron los genes Cg0120 (tioesterasa), el Cg1021 (lipasa o esterasa), el Cg1294 ( $\alpha / \beta$-hidrolasa) y el Cg2528 (carboxilesterasa), para obtener enzimas pertenecientes a las diferentes familias de la superfamilia de las esterasas.

Las colonias seleccionadas se inocularon en medio LB (Luria Broth), en las condiciones descritas en el apartado de material y métodos. Como resultado de la expresión de cada gen se obtuvieron las proteínas recombinantes en forma de cuerpos de inclusión.

Para intentar solubilizarlas, se pusieron distintos cultivos a distinta densidad óptica $(0,2$ y 0,7$)$, para inducirlos a diferentes temperaturas $\left(18{ }^{\circ} \mathrm{C}\right.$, $20^{\circ} \mathrm{C}$ y $25^{\circ} \mathrm{C}$ ) y con distintas concentraciones de IPTG $(0,2 \mathrm{mM}, 0,5 \mathrm{mM}, 1 \mathrm{mM}$ y $2 \mathrm{mM})$, optimizándose así la expresión de la proteína en forma soluble en algunos casos.

La proteína recombinante que codificaba el gen $\mathrm{Cg} 0120$ se solubilizó, induciendo el cultivo a $20{ }^{\circ} \mathrm{C}$, durante 12 horas, con una concentración de IPTG de $0,5 \mathrm{mM}$. La actividad esterasa se midió con $p$-nitrofenilacetato, la actividad lipasa con $p$ nitrofenillaureato y la actividad tioesterasa con el S-metiltiobutanoato. No se observó actividad.

La proteína recombinante que codificaba el gen Cg1294 se consiguió solubilizar parcialmente, 


\begin{tabular}{clccc}
\hline Gen & \multicolumn{1}{c}{$\begin{array}{c}\text { Actividad } \\
\text { Predicha }\end{array}$} & $\begin{array}{c}\mathbf{N}^{\circ} \text { de } \\
\text { aminoácidos }\end{array}$ & Peso molecular kDa & Estudiada \\
\hline Cg0120 & Esterasa, lipasa tíoesterasas & 259 & 27,8 & No clonada \\
Cg0153 & Esterasa/lipasa & 308 & 32,3 & No clonada \\
Cg1021 & Esterasa/lipasa & 177 & 19,1 & No clonada \\
Cg1104 & Proteína de membrana o esterasa & 426 & 45,6 & No clonada \\
Cg1284 & Carboxilesterasa & 559 & 62,3 & No clonada \\
Cg1294 & Esterasa o a/ß-hidrolasa & 298 & 33,15 & No clonada \\
Cg1572 & Putativa fosfodiesterasa & 235 & 25,2 & No clonada \\
Cg1873 & Acil-CoA tioesterasa & 282 & 31,7 & No clonada \\
Cg2528 & Carboxilesterasa & 393 & 43,6 & No clonada \\
Cg2692 & Tioesterasa & 155 & 17,2 & No clonada \\
Cg2768 & a-aminopeptidasa o D-esterasa & 304 & 30,2 & No clonada \\
Cg3194 & Fosfoesterasa & 224 & 24,2 & No clonada \\
Cg3215 & Putativa fosfodiesterasas & 253 & 28,7 & No clonada \\
Cg3393 & Putativa fosfoesterasas & 1461 & 157,037 & No clonada \\
\hline
\end{tabular}

Tabla 1. Esterasas hipotéticas de Corynebacterium glutamicum ATTC 13032.

Table 1. Putatives esterases from Corynebacterium glutamicum ATTC 13032

induciendo el cultivo a $20^{\circ} \mathrm{C}$, durante 12 horas, con una concentración de IPTG de $0,5 \mathrm{mM}$. Se midió la actividad esterasa con $p$-nitrofenilacetato, la actividad lipasa con $p$-nitrofenillaureato y la actividad patatina con $p$-nitrofenillaureato en forma de micelas mixtas. Las micelas mixtas se prepararon siguiendo el método descrito por Jiménez et al. (2001). No se detectó actividad.

La proteína recombinante que codificaba el gen $\mathrm{Cg} 1021$ no se pudo solubilizar induciendo el cultivo a $20^{\circ} \mathrm{C}$, durante 12 horas, con una concentración de IPTG de $0,5 \mathrm{mM}$. Con el fin de obtenerla soluble, se clonó el gen en los vectores de expresión que expulsan la proteína al espacio periplasmático de la célula, pET-26b+ y pET-40b+. La expresión de la proteína se hizo a distintas temperaturas $\left(18{ }^{\circ} \mathrm{C}, 20^{\circ} \mathrm{C}\right.$ y $\left.25^{\circ} \mathrm{C}\right)$ a baja densidad óptica $(0,2)$ y bajas concentraciones de IPTG $(0,5$, y $0,2 \mathrm{mM}$ ). Finalmente, la proteína no se solubilizó, quedando en forma de cuerpos de inclusión.

La proteína recombinante que codificaba el gen $\mathrm{Cg} 2528$, también se expresó formando cuerpos de inclusión cuando se indujo a $30^{\circ} \mathrm{C}$, pero se solubilizó, parcialmente, induciendo el cultivo a $20{ }^{\circ} \mathrm{C}$, durante 8 horas, con una concentración de IPTG de $0,5 \mathrm{mM}$. Una vez solubilizada, mediante sonicación, se consiguió el extracto enzimático, usándose para medir las actividades esterasa el $p$ nitrofenilacetato, lipasa el $p$-nitrofenillaureato y tioesterasa el S-metiltiobutanoato, presentando actividad esterasa y tioesterasa. A esta esterasa se le denominó Est2528.

Tras optimizar la expresión de la Est2528, se procedió a realizar cultivo de alta densidad en el medio TB, en el cual las células se indujeron con $0,5 \mathrm{mM}$ de IPTG, durante 8 horas a $20^{\circ} \mathrm{C}$.

En la figura 1 puede observarse que la Est2528 representa el $16 \%$ de todas las proteínas que están en la fracción soluble, y la que no se logró solubilizar representa el $36 \%$ de la proteína de la fracción insoluble. Si consideramos la suma de las dos bandas de la Est2528, la que está en forma activa y la que está formando cuerpos de inclusión, el $100 \%$, se consiguió solubilizar el $40 \%$ y $60 \%$ restante siguió formando cuerpos de inclusión.

La Est2528 se purificó con sulfato amónico. Se alcanzó, al precipitar la proteína al $40 \%$ del sulfato amónico, una actividad específica de 158 $\mathrm{UE} / \mathrm{mg}$ de proteína.

La determinación del peso molecular por electroforesis desnaturalizante en SDS-PAGE (fig. 1) resulta 45,8 $\mathrm{kDa}$ para el monómero, mientras que el peso molecular de la enzima nativa estimado por filtración en gel resultó ser de 44,9 kDa. Como el peso molecular teórico de la secuencia de aminoácidos es $44,6 \mathrm{kDa}$, se deduce que la enzima es un monómero en su forma nativa.

\section{Características cinéticas}

La actividad enzimática se midió espectrofotométricamente usando ésteres de $p$-nitrofenoles como sustratos.

La figura 2 representa el porcentaje relativo de actividad expresada sobre el $p$-nitrofenilacetato, el $p$-nitrofenilbutirato y el $p$-nitrofenilcaprilato (sobre el $p$-nitrofenilmiristato y $p$-nitrofenillaureato no presentó actividad) a diferentes pHs. Como puede observarse en la figura 2 , en todos los casos 


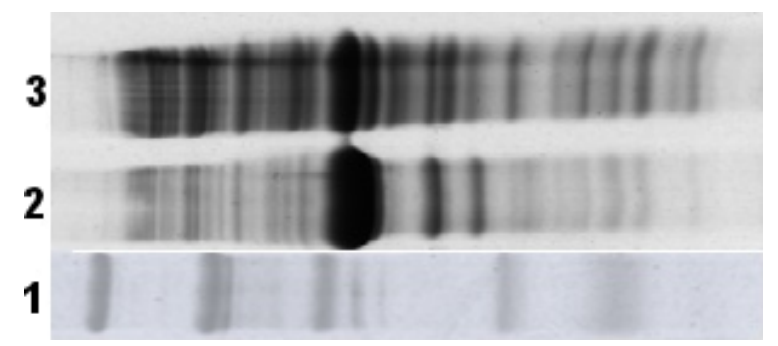

Figura 1. SDS-PAGE de la Est2528. Cada calle contiene $5 \mu \mathrm{g}$ de proteína. Calle 1: Marcador de proteínas (de izquierda a derecha: $175 \mathrm{kDa} \beta$-galactosidasa; $83 \mathrm{kDa}$ miosina; $62 \mathrm{kDa}$ glutamato deshidrogenasa; $47,5 \mathrm{kDa}$ aldolasa; $32,5 \mathrm{kDa}$ triosa fosfato isomerasa; $25 \mathrm{kDa} \beta$-lactoglobulina; $16,5 \mathrm{kDa}$ lisocima). Calle 2: Fracción insoluble. Calle 3: Fracción soluble.

Figure 1. SDS-PAGE of the Est2528. Each lane contains $5 \mu \mathrm{g}$ protein. Lane 1: Molecular weight markers (from left to right: $175 \mathrm{kDa} \beta$-galactosidase; $83 \mathrm{kDa}$ myosine; $62 \mathrm{kDa}$ glutamate dehydrogenase; $47.5 \mathrm{kDa}$ aldolase; $32.5 \mathrm{kDa}$ triosephosphate isomerase; $25 \mathrm{kDa} \beta$-lactoglobuline; $16.5 \mathrm{kDa}$ lysozyme); Lane 2: insoluble fraction; Lane 3: soluble fraction.

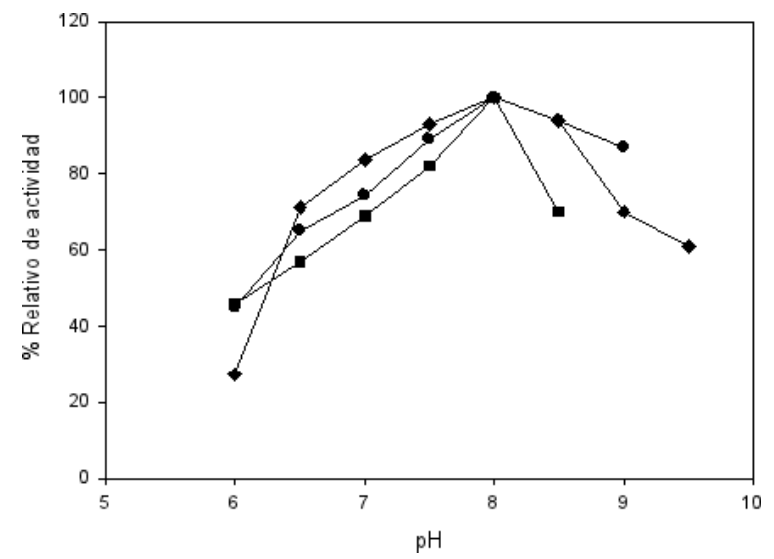

Figura 2. Efecto del pH sobre la actividad de la Est2528. Los sustratos utilizados fueron $p$-nitrofenilcaprilato $(\diamond), p$-nitrofenilbutirato $(\bullet), p$-nitrofenilacetato $(\boldsymbol{\bullet})$. Todas las medidas de actividad ha sido realizadas bajo las condiciones estándares, excepto para los parámetros estudiados.

Figure 2. Effect of $\mathrm{pH}$ on Est2528 activity. Substrates used were $p$-nitrophenyl caprilate $(\bullet), p$-nitrophenyl butirate $(\bullet), p$-nitrophenyl acetate (-). The esterase activity was measured in all cases in standard assay conditions, except for the tested parameter.

presenta la máxima actividad a $\mathrm{pH}$ 8. Este dato concuerda con el de otras esterasas como la EstGtA2 (Charbonneau et al. 2010), la Sm23 (Hwang et al. 2010) o la esterasa de Sulfolobus tokodaii (Suzuki et al. 2004).

El efecto de la temperatura sobre la actividad catalítica se determinó usando el $p$-nitrofenilbutirato y el tampón Tris- $\mathrm{HCl} 100 \mathrm{mM}$ a $\mathrm{pH}$ 8. Las temperaturas ensayadas fueron de $\operatorname{los} 20^{\circ} \mathrm{C}$ a los $60{ }^{\circ} \mathrm{C}$. Los resultados ponen en evidencia que la temperatura a la cual presenta una mayor actividad la enzima es a $40{ }^{\circ} \mathrm{C}$, y presenta un $20 \%$ me- nos de actividad a $35^{\circ} \mathrm{C}$ y a $45^{\circ} \mathrm{C}$ (datos no mostrados)

La termoestabilidad de la Est2528 se investigó a diferentes temperaturas, de $42{ }^{\circ} \mathrm{C}$ a $60^{\circ} \mathrm{C}$, y a diferentes tiempos de incubación. Cuando se incubó la enzima a $60{ }^{\circ} \mathrm{C}$, ésta quedó inactiva en menos de 5 minutos. Al incubarla a $55^{\circ} \mathrm{C}$ la enzima perdió el $95 \%$ de su actividad a los 5 minutos y a 50 ${ }^{\circ} \mathrm{C}$ perdió al $90 \%$ de su actividad a los 5 minutos y a los 15 minutos perdió el $100 \%$. Cuando se incubó a $45^{\circ} \mathrm{C}$, perdió a los 5 minutos el $45 \%$ de su actividad, y el resto la fue perdiendo de forma progresiva hasta quedar casi inactiva a los $50 \mathrm{mi}$ nutos. Y por último, se incubó a $42{ }^{\circ} \mathrm{C}$ donde se observó que la enzima pierde el $50 \%$ de su actividad a los 25 minutos y a los 80 minutos de incubación queda con tan solo el $18 \%$ de su actividad inicial (datos no mostrados). Estos datos muestran que la Est2528 no es una enzima termoestable, como son la EstGtA2 (Charbonneau et al. 2010), la Est3 de Sulfolobus solfataricus (Kim \& Lee 2004) o la esterasa de $S$. tokodaii (Suzuki et al. 2004).

\section{Efecto de los detergentes y de los disolventes orgánicos sobre la Est2528}

Las esterasas y lipasas se utilizan ampliamente en la industria alimentaria como ingredientes o aditivos (Figueroa-Espinosa \& Villeneuve 2005; Chevil et al. 2006) para la transformación de aceites y grasas (Bloomer et al. 1990; Undurraga et al. 2001), para el desarrollo de aromas, para la síntesis de antibióticos (Sánchez-Ferrer et al. 2007), la separación de mezclas puras (Bournscheuer \& Kazlauskas 1999; Reetz 2004) y fabricación de detergentes etc. En todos estos procesos se requiere que la enzima utilizada resista la presencia de detergentes y disolventes orgánicos. Por lo que se estudió su efecto sobre la enzima.

Se evaluaron el SDS, el Tween 20 y el Triton X-100 al $5 \%$ y al $10 \%$. Se observó que el SDS tiene un efecto desnaturalizante inmediato sobre la enzima y que con el Triton X-100 la enzima pierde el $45 \%$ de su actividad si se emplea al $5 \%$, ya que al $10 \%$ se pierde completamente la actividad. Con el Tween 20 la actividad relativa de la enzima al $5 \%$ es del $32 \%$ y cuando alcanza el $10 \%$ del detergente la Est2528 retiene tan solo el $26 \%$ de la actividad. Comparando los resultados obtenidos con los de esterasas termoestables como son la Est3 (Kim \& Lee. 2004) y la Sm23 (Hwang et al. 2010), la Est2528 es más estable en presen- 
cia de detergentes que la Sm23, mientras que en el caso de la Est3 es más estable que la Est2528 solo en presencia de Triton X-100, lo que indica que la Est2528 es una buena candidata para su posible aplicación industrial.

Del estudio llevado a cabo con los disolventes orgánicos se observa que con todos los usados (etanol, propanol, metanol, DMSO, acetona y acetonitrilo) al $5 \%(\mathrm{~V} / \mathrm{V})$ la enzima es estable. Con un $10 \%$ de DSMO la Est2528 no pierde actividad y al alcanzar el $20 \%$ es cuando empieza a tener pérdidas significativas con todos los disolventes, siendo más estable con el metanol y el DMSO (Tabla 2). El acetonitrilo y el propanol son los que más pérdida de actividad provocan en la enzima, inactivándola cuando se añaden al $20 \%$. Y el etanol y la acetona provocan mermas significativas de actividad al $20 \%$, llegando a inactivarla cuando están al $30 \%$.

Si se comparan los datos de actividad residual con la Est3 (Kim \& Lee. 2004) y la Sm23 (Hwang et al. 2010), se pone de manifiesto que la Est2528 es mucho más estable, lo que puede hacerla más atractiva industrialmente, ya que a la temperatura a la cual se utilizan las esterasas en algunos procesos tecnológicos las proteínas mesófilas tiene una mayor actividad que las proteínas termófilas. La ventaja que presentan la mayoría de las proteínas termoestables frente a las mesófilas, es su resistencia a desnaturalizarse en condiciones extremas como es el exponerlas a elevadas concentraciones de disolventes orgánicos. Por lo que, la Est2528 presenta la ventaja de ser estable en presencia de elevadas concentraciones de disolventes orgánicos y la temperatura a la que presenta su máxima actividad está entre el rango de temperatura a las que se trabaja a nivel industrial (Mnisi et al. 2005).

\section{Efecto de los cationes sobre la Est2528}

Se evaluó el efecto de los iones y aniones sobre la actividad enzimática. La presencia de mercurio inhibe completamente la enzima, siendo el mercurio un inhibidor de grupos tioles ( $\mathrm{SH}$ ), lo que puede indicar que alguna cisteína está involucrada en la actividad enzimática (Kim \& Lee 2004).

El calcio produce perdidas del $76 \%$ y la inhibición es irreversible, ya que no se recupera con la presencia del EDTA. Este dato es llamativo, ya que se ha referenciado en la bibliografía que es un activador de algunas esterasas (Nawani et al. 2006). Un ejemplo sería la Est53 de Thermotoga marítima que requiere la presencia de calcio y magnesio, para incrementar su actividad más del doble (Kakugawa et al. 2007).

Con el níquel, la pérdida de actividad es del $42 \%$, pero, al añadir EDTA, recupera parte de ella y tan solo se pierde el $24 \%$. El manganeso también produce pérdidas significativas del orden del $70 \% \mathrm{y}$, al añadirle EDTA, tan solo se recupera un $7 \%$. El aluminio produce mermas del $21 \%$, que se contrarrestan al añadirle EDTA. Sodio, litio y potasio no tienen ningún efecto sobre la enzima. El magnesio también provoca pérdidas de actividad del $42 \%$ en la Est2528 (datos no mostrados)

\begin{tabular}{|c|c|c|c|c|}
\hline \multicolumn{5}{|c|}{ Actividad (\%) } \\
\hline Disolvente & $5 \%(v / v)$ Solvente & $10 \%(v / v)$ Solvente & $20 \%(v / v)$ Solvente & $40 \%(v / v)$ Solvente \\
\hline Etanol & 100 & 92 & 75 & 0 \\
\hline Metanol & 100 & 90 & 88 & 57 \\
\hline Acetonitrilo & 100 & 95 & 56 & 0 \\
\hline Acetona & 100 & 93 & 71 & 0 \\
\hline DMSO & 100 & 90 & 74 & 45 \\
\hline Propanol & 100 & 90 & 58 & 0 \\
\hline Inhibidor & & $0.5 \mathrm{mM}$ & $5 \mathrm{mM}$ & \\
\hline DEPC & & 91 & 76.7 & \\
\hline PGO & & 93 & 95 & \\
\hline PMFS & & 100 & 95 & \\
\hline Pepstatina A & & 71 & 49 & \\
\hline PLP & & 53 & 51 & \\
\hline Paraoxón & & 3.8 & 0 & \\
\hline Eserina & & 80 & 0 & \\
\hline
\end{tabular}

Tabla 2. El efecto de los diferentes moduladores y solventes orgánicos sobre la actividad de la Est2528.

Table 2. Effect of different modulators and organic solvents on Est25281 activity. 


\section{Aminoácidos esenciales para la catálisis}

Para poder investigar los aminoácidos que están implicados en el mecanismo de la catálisis enzimática, se incubó la esterasa Est2528 con los reactivos inhibidores específicos de aminoácidos durante 30 minutos a $30{ }^{\circ} \mathrm{C}$ a dos concentraciones distintas $(0,5 \mathrm{mM}$ y $5 \mathrm{mM})$.

El DEPC, que es un inhibidor específico de histidinas, inhibe a la enzima un $23 \%$ a la concentración de $5 \mathrm{mM}$. El PMFS, que es el inhibibidor especifico de serinas, inhibe la enzima en torno al $50 \%$ a la mayor concentración utilizada, de gual forma que ocurre con el piridoxal, que es el inhibidor especifico de lisinas y el PGO (inhibidor especifico de arginina) y la pepstatina-A, que es un inhibidor especifico de ácido aspártico, producen una pérdida de actividad del $5 \%$ a una concentración de $5 \mathrm{mM}$ (Tabla 2). Estos datos sugieren que los aminoácidos del centro activo no son muy accesibles a estos inhibidores, motivo por el cual, no inhiben la actividad enzimática totalmente, sino parcialmente.

Por otro lado, se ha usado el paraoxón y la eserina para clasificar la enzima. Y, aunque no queda inhibida completamente con el PMFS, sí con el paraoxon, que es otro compuesto organofosforado que inhibe la serina, por lo que la enzima puede considerarse una serinesterasa.

El hecho de que la enzima quede completamente inhibida en presencia de paraoxon y eserina (amina terciaria inhibidora de acetilcolinesterasas), sugeriría que la enzima se pueda considerar como una colinesterasa (Park et al., 2006); por lo que se le midió tanto la actividad colinesteresa como la actividad butirilesterasa, y no presentó actividad sobre ninguno de los dos sustratos.

\section{Parámetros cinéticos de la esterasa Est2528}

Se determinaron los parámetros cinéticos, Km y Vmax, a partir de las curvas de velocidad frente a la concentración de sustrato mediante regresión no lineal a la ecuación de Michaelis-Menten, utilizando el programa Sigmaplot (SPSS, Germany).

La concentración final de enzima utilizada en cada medida fue de 0,07 UE ( $p$-nitrofenilacetato) en un mililitro de reacción a su pH óptimo, para todas las medidas y con todos los sustratos.

La actividad esterasa de la enzima se midió con dos familias de sustratos, los $p$-nitrofenilos ( $p$-nitrofenilacetato, $p$-nitrofenilbutirato y $p$-nitrofenilcaprilato), y la de $\operatorname{los} \alpha$ y $\beta$-naftilos ( $\alpha$-naftil acetato, $\beta$-naftil acetato, $\alpha$-naftil butirato y $\beta$-naftil butirato).

Para el $p$-nitrofenilacetato, la $\mathrm{Km}$ de la enzima es de 2,5 mM y la Vmax de $70 \mu \mathrm{M} / \mathrm{min}$. Para el $p$ nitrofenilbutirato la $\mathrm{Km}$ es de $681 \mu \mathrm{M}$ y la Vmax de $129 \mu \mathrm{M} / \mathrm{min}$. Para el $p$-nitrofenilcaprilato la $\mathrm{Km}$ es de $113 \mu \mathrm{M}$ y la Vmax de 9,2 $\mu \mathrm{M} / \mathrm{min}$. Los datos obtenidos de las $\mathrm{Km}$ nos indican que la Est2528 presenta una mayor afinidad sobre el $p$ nitrofenilcaprilato, pero si nos basamos en la eficacia (calculada Vmax/Km) se observa que se decanta hacia el $p$-nitrofenilbutirato.

Para el $\alpha$-naftil acetato los valores de $\mathrm{Km}$ y Vmax son $275 \mu \mathrm{M}$ y $36,6 \mu \mathrm{M} / \mathrm{min}$, respectivamente, los del $\beta$-naftil acetato son $604 \mu \mathrm{M}$ y 42,9 $\mu \mathrm{M} / \mathrm{min}$, los del $\alpha$-naftil butirato son $122 \mu \mathrm{M}$ y $23,2 \mu \mathrm{M} / \mathrm{min}$ y los del $\beta$-naftil butirato son 192 $\mu \mathrm{M}$ y $24 \mu \mathrm{M} / \mathrm{min}$. Los datos de Km ponen de manifiesto que la enzima presenta más afinidad por $\operatorname{los} \alpha$-naftil acetato y $\beta$-naftil acetato que por el $\alpha$ naftil o el $\beta$-naftil butirato. En cuanto a la eficacia, esta es mayor sobre la posición $\alpha$ que la $\beta$ y sobre el naftil acetato que sobre el butirato.

La Est2528 presentó esta actividad tioesterasa, con $\mathrm{Km}$ de 9,9 mM y Vmax 51,7 $\mu \mathrm{M} / \mathrm{min}$.

La actividad tioesterasa presente en algunas esterasas como la EST2 de Alicyclobacillus acidocaldarius (Mandrich et al. 2006) se ha relacionado con su intervención en el proceso de maduración del queso. Por tanto, la presencia de actividad tioesterasa en la Est2528 pone de manifiesto su posible aplicación industrial en la maduración de los quesos como coadyuvante tecnológico.

\section{Modelado estructural de la esterasa Est2528}

Para conocer la homología que presenta la Est2528 con otras esterasas bacterianas, se realizó un BLAST en la base de datos del Nacional Center for Biotechnology Information (NCBI: http:// www.ncbi.nlm.nih.gov/).

Los resultados del análisis no ofrecieron mucha información acerca de la esterasa, ya que la proteína presentaba una elevada homología (98\% al $45 \%$ ) con otras esterasas hipotéticas de distintas especies de Corynebacterium, aun sin estudiar.

A pesar de no ofrecer ningún parecido con ninguna esterasa estudiada, según los resultados del BLAST, se envió la secuencia de aminoácidos de la Est2528 a Expert Protein Análisis System Proteomic Server (http://www.expasy.ch/swissmod/ SWISS-MODEL.html) para la creación del modelo tridimensional teórico de la esterasa. Para mo- 
delarla, el programa informático usó como molde la Est55 (2OGS) de Geobacillus stearothermophilus (Liu et al. 2007), con la que muestra un $23 \%$ de homología. Esta homología ha permitido identificar las similitudes estructurales, asignar la posición de la triada catalítica y la construcción del modelo tridimensional.
En la figura 3 podemos ver el alineamiento de aminoácidos de ambas proteínas y la estructura secundaria predicha, formada por 12 láminas $\beta \mathrm{y}$ 10 hélices $\alpha$ más grandes y 4 más pequeñas. Los aminoácidos que son iguales en ambas aparecen sombreados de rojo y los que son de la misma familia aparecen escritos con rojo.
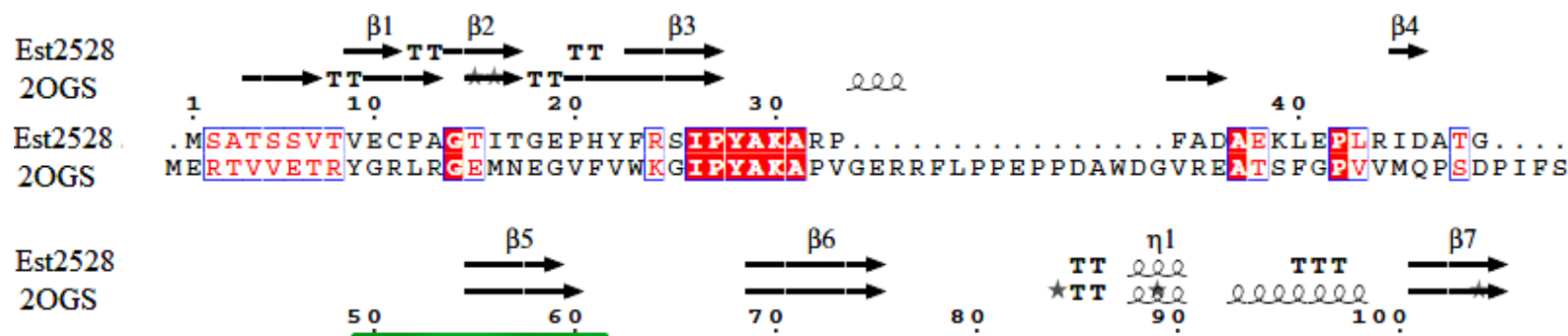

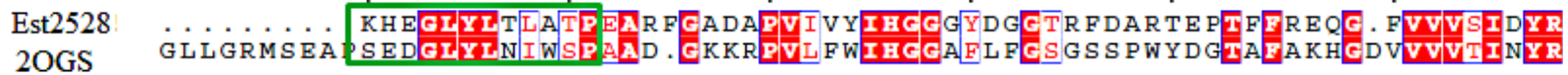

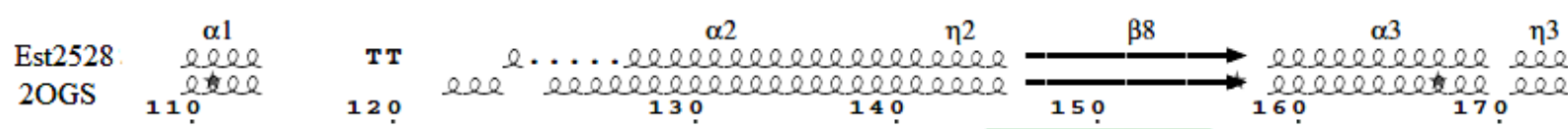

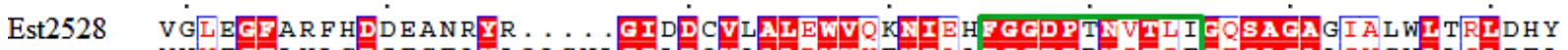
2OGS

Est2528

2OGS

Est 2528

2OGS

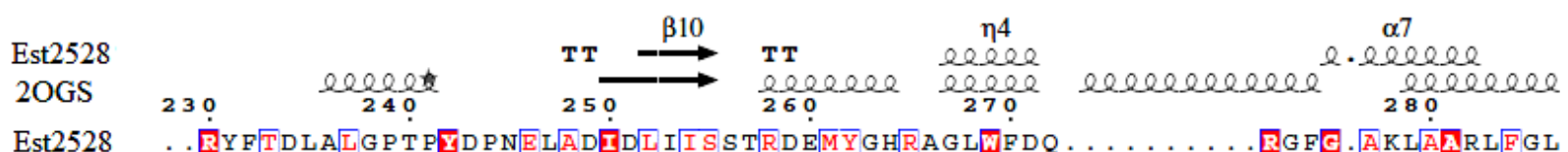

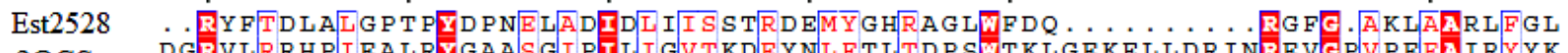
2OGS

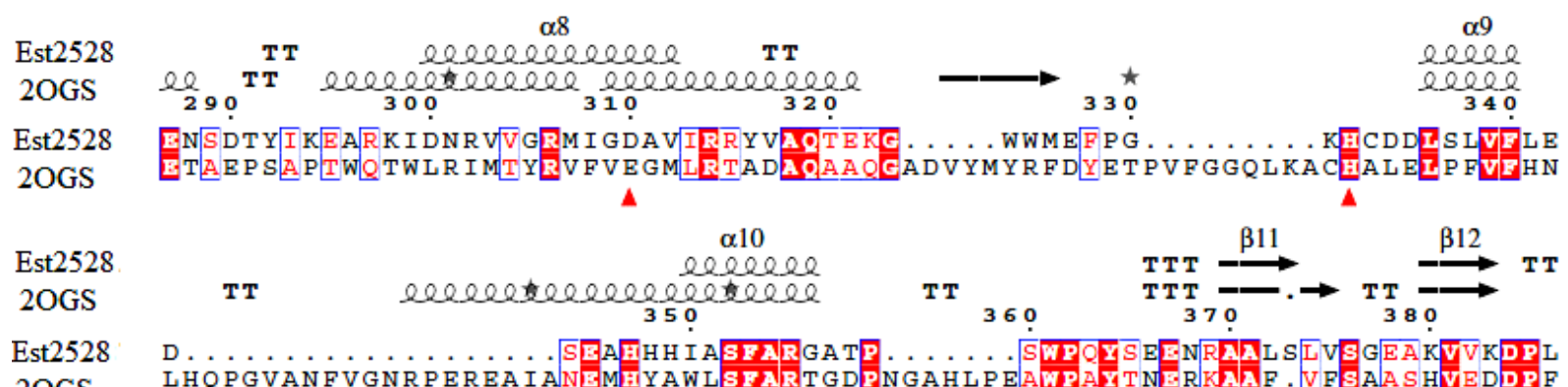

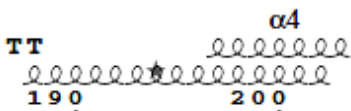

eelee
ele
10

eqRelere 220 $\quad$ *

FERQPFARKG

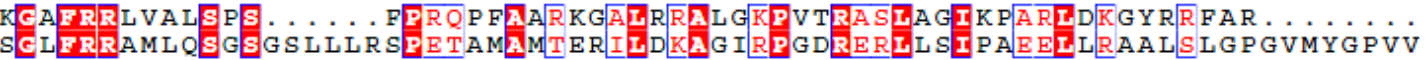
DGRVLRRHPIEALRYGAAGGIPILIGVTKDEYNLETLTDP SWTKLGEKELLDR INREVGPVPEEAIRYYK

\section{Est2528 \\ 2OGS \\ Est 2528 \\ $20 \mathrm{OG}$}

Figura 3. Alineamiento de los aminoácidos de la carboxilesterasa de Corynebacterium glutamicum (Est2528) con la carboxilesterasa 2OGS de Geobacillus stearothermophilus. Los símbolos encima de los bloques de las secuencias de aminoácidos representan la estructura secundaria, resortes representan hélices $\alpha$, y las flechas representan las láminas $\beta$. Los residuos que forman el bolsillo de especificidad hidrófobo se indican con asteriscos negros pequeños. Los triángulos representan la localización del sitio activo.

Figure 3 Sequence aligment for Est2528 from Corynebacterium glutamicum with carboxylesterase 2OGS from Geobacillus stearothermophilus. Symbols above blocks of sequences represent the secondary structure, springs represent helices, and arrows represent $\beta$ strands. The residues forming the hydrophobic specificity pocket are indicated by small black asterisks. Triangles represent the location of the active site. 
Los aminoácidos del centro activo de la Est2528 están señalados con un triángulo rojo. Son la serina 158 , el aspártico 310 y la histidina 332 , situados en la misma posición que el centro activo de la 2OGS, con la diferencia de que el ácido aspártico de la Est2528 es sustituido por el ácido glutámico en la 2OGS.

La serina catalítica está localizada en el pentapéptido GQSAG, donde la glutamina (Q) de la Est2528 es sustituida por el ácido glutámico en la $2 \mathrm{OGS}$.

El rectángulo verde rodea las secuencias de aminoácidos cercanas al $\mathrm{N}$-terminal que aparecen conservados en todas las carboxilesterasas de tipo B (Prim et al. 2000). La secuencia de aminoácidos del N-terminal presenta una mayor homología que los que están en el C-terminal.

Comparando el modelado tridimensional (fig. 4A) de la Est2528 con el de la carboxilesterasa
2OGS (Liu et al. 2007), se observa que presenta la estructura típica de las $\alpha / \beta$-hidrolasas, formando una estructura central formada por hojas $\beta$ interconectadas con hélices $\alpha$, y que se pliega formando 3 dominios bien caracterizados, el dominio regulatorio, el dominio catalítico y un dominio alfa/beta adicional.

El centro activo de la Est2528 se ha señalizado de azul la serina, de magenta el aspártico y de narnaja la histidina. Y en la Est2528 se ve que los 3 aminoácidos se encuentran situados en el bolsillo catalítico en la misma posición que en la 2OGS (Liu et al. 2007).

Los diagramas topológicos de ambas esterasas (fig. 4B), dan una visión más simplificada de la estructura tridimensional. Así, se observa la estructura típica de las $\alpha / \beta$-hidrolasas, formada por una estructura central de hojas $\beta$ (triángulos azules) interconectadas con hélices $\alpha$ (círculos rojos).
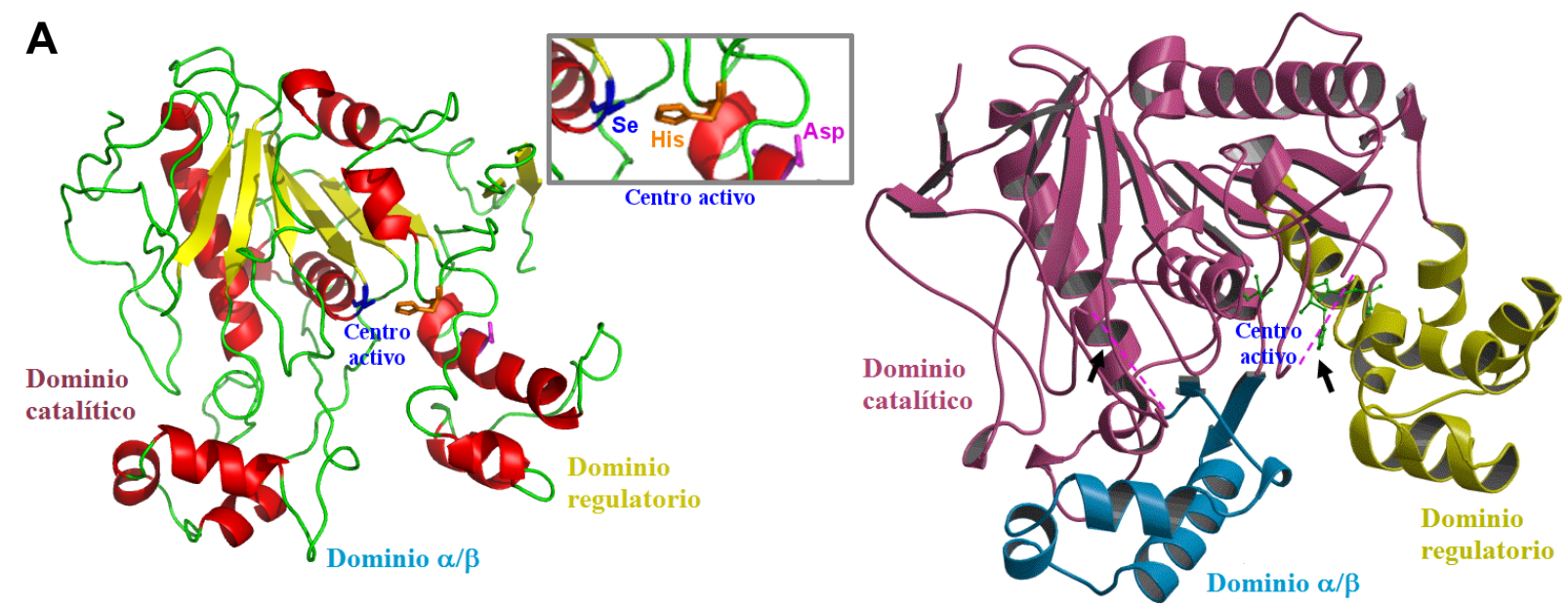

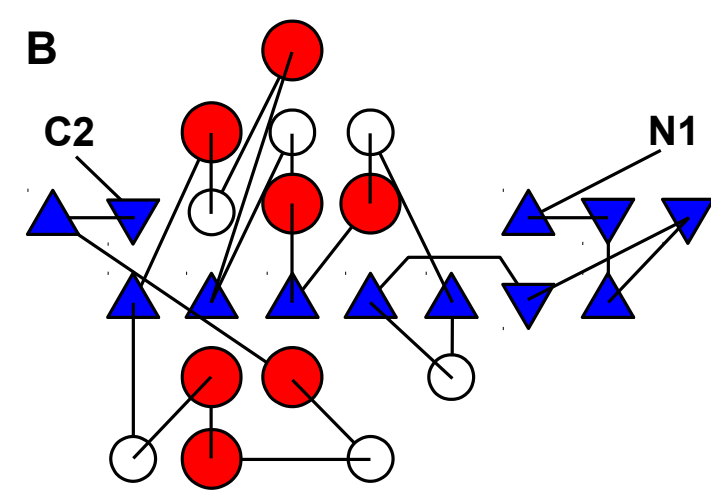

Est2528

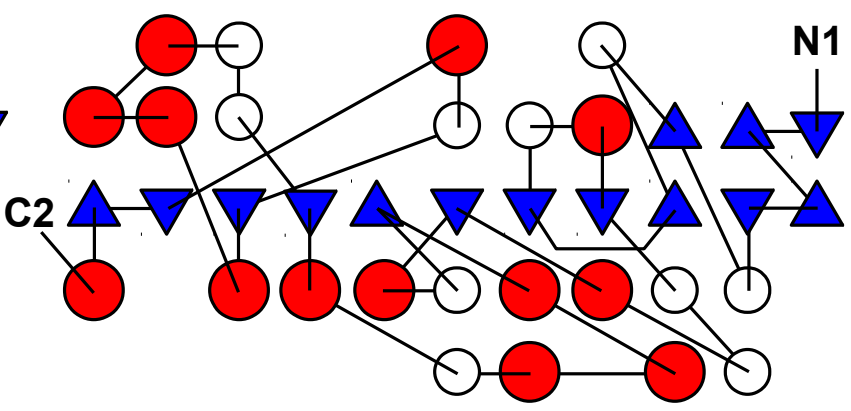

2OGS

Figura 4. A. Modelado de la carboxilesterasa de Corynebacterium glutamicum Est2528 y estructura de la carboxilesterasa cristalizada de Geobacillus stearothermophilus (2OGS). B. Diagrama topológico de la Est2528 y 2OGS. Las hélices $\alpha$ están representadas por círculos, las láminas $\beta$ por triángulos y otras hélices $\alpha$ pequeñas de la estructura por círculos blancos.

Figure 4 A. Schematic representation of tertiary structure of Est2528 from Corynebacterium glutamicum and 2OGS from Geobacillus stearothermophilus. B. Topology diagram of Est2528 and 2OGS. $\alpha$-helices are represented by circles and $\beta$-strands by triangles. White circles represent other small helices in the structure. 
Concretamente, la Est 2525 tiene 7 hojas $\beta$ paralelas entre ellas excepto una que es antiparalela, 7 hélices $\alpha$ principales y 6 secundarias. Mientras que la 2OGS tiene 11 hojas $\beta$ (3 antiparalelas y 8 paralelas), 13 hélices $\alpha$ principales y 10 hélices $\alpha$ secundarias.

La diferencia que se aprecia en el número de hélices $\alpha$ grandes y pequeñas entre la figura 3 y la figura $4 \mathrm{~B}$, es debida a que cada programa bioinformático tiene diferentes criterios para adjudicar las hélices $\alpha$ grandes o pequeñas. Así, las $\alpha 5$ y $\alpha 9$ de la figura 3 son consideradas hélices grandes y en la figura $4 \mathrm{~B}$ como hélices pequeñas, otra diferencia es que $\eta 2$ de la figura 3 , en la figura $4 B$ no la representa porque incluye toda la secuencia de aminoácidos dentro de la hélice $\alpha 2$.

Ambas esterasas presentan una región de hojas $\beta$ en el extremo $\mathrm{N}$-terminal, antes de generarse la estructura central y sólo en la Est2528 vuelve a presentar hojas $\beta$ en el extremo C-terminal.

El 23\% de la homología de la esterasa Est2528 con la $2 \mathrm{OGS}$ de $G$. stearothermophilus indicaría que de acuerdo a la clasificación de Arpigny \& Jaeger (1999), esta enzima pertenecería a la familia VII, que presentan una elevada homología (30\% de identidad y $40 \%$ de similitud) con acetilcolinesterasas de eucariotas y carboxilesterasa del intestino e hígado.

\section{Conclusión}

En este articulo ha sido descrita la clonación de varias putativas esterasas de C. glutamicum, pudiendo clonar, sobreexpresar, caracterizar cinéticamente y modelar, solo una, la Est2528, codificada por el gen $\mathrm{Cg} 2528$. La enzima presentó su máxima actividad a $\mathrm{pH} 8$, a $40{ }^{\circ} \mathrm{C}$ y sobre ésteres de cadena corta, motivo por el cual es una esterasa. El hecho de que la Est2528 presente una gran estabilidad en presencia de disolventes orgánicos, la hace muy atractiva para su posible aplicación industrial.

Basándose en la secuencia de aminoácidos y al modelado estructural, puede deducirse el centro activo de la enzima esta formado por la serina 158, histidina 332 y aspártico 332.

\section{Referencias}

Arpigny JL \& Jaeger KE. 1999. Bacterial lipolytic enzymes: classification and properties. The Biochemi- cal Journal 343, 177-183.

Bloomer S, Adlercreutz P \& Mattiasson B. 1990. Triglyceride interesterification by lipase. 1. Cocoa butter equivalents from a fraction of palm oil. Journal of the American Oil Chemists' Society 67(8): 519-524.

Bornscheuer UT \& Kazlauskas R.J. 1999. Hydrolase in organic synthesis: Regio- and stereoselective biotransformations. Wiey-VCH, Weinheim.

Charbonneau DM, Meddeb-Mouelhi F \& Beauregard M. 2010. A novel thermostable carboxylesterase from Geobacillus thermodenitrificans: evidence for a new carboxylesterase family. The Journal of Biochemistry 148: 299-308.

Chevil L, Humeau C, Falcimaigne A, Engasser JM \& Ghoul M. 2006. Enzymatic acylation of flavonoids. Process Biochemistry 41: 2237-2251.

Figueroa-Espinosa MC \& Villeneuve P. 2005 Phenolic acid enzymatic lipophilization. Journal of Agriculture and Food Chemistry 53: 2779-2787.

Hwang H, Kim S, Yoon S, Ryu Y, Lee SY \& Kim TD. 2010. Characterization of a novel oligomeric SGNHarylesterase from Sinorhium meliloti 1021. International Journal of Biological Macromolecules 46: 1451452.

Jiménez M, Escribano J, Pérez-Gilabert M, Chazarra S, Cabanes J \& García-Carmona F. 2001. An Octaethylene Glycol Monododecyl Ether-Based Mixed Micellar Assay for Determining the Lipid Acyl Hydrolase Activity of Patatin. Lipids 36: 1169-1174.

Kakugawa S, Fushinobu S, Wakagi T \& Shoun H. 2007. Characterization of a thermostable carboxylesterase from the hyperthermophilic bacterium Thermotoga maritima. Applied Microbiology and Biotechnology 74: 585-591.

Kalinowski J, Bath B, Bartels D, Bischoff M., Bott M, Burkovski A, Dusch N, Eggeling L, Eikmanns J, Gaigalat L, Goesmann A, Hartmann M, Huthmacher K, Krämer R, Linke B, McHardy C, Meyer F, Möckel B, Pfefferle W, Pühler A, Rey DR, Úckert C, Rupp O, Sahm H, Wendisch V, Wiegräbe I \& Tauch A. 2003 The complete Corynebacteerium glutamicum ATCC 13032 genome sequence and its impact on the production of L-aspartate-derived amino acids and vitamins. Journal of Biotechnology 104: 5-25.

Kim S \& Lee S. 2004. Thermostable esterase from a Thermoacidophilic Archaeon: Purification and Characterization for Enzymatic Resolution of a Chiral Compound. Bioscience Biotechnology and. Biochemistry 68: 2289-2298.

Kinoshita S, Udaka S \& Shimono M. 1957. Studies on the amino acid fermentation. I. Production of I-glutamic acid by various microorganisms. Journal of General and. Applied. Microbiology 3: 193-205.

Laemmli U.K. 1970. Cleavage of structural proteins during the assembly of the head of bacteriophage T4. Nature 227: 680-685.

Leuchetenberger W. 1996. Amino acids-technical production and use. In: Rehm, H.-J., Reed, G., Pühler, A., Stadler, P. Eds., Biotechnology, vol. 6. VCH, Weinheim, Germany, pp. 465-502.

Liu P, Ewis H, Tai P, Li CD \& Weber I. 2007. Crystal structure of the Geobacillus stearothermophilus car- 
boxylesterase Est55 and its activation of prodrug CPT-11. Journal of Molecular Biology 16: 212-223.

Lozada-Ramírez JD, Martínez-Martínez I, Sánchez-Ferrer A \& García-Carmona F. 2006. A colorimetric assay for S-adenosylhomocisteine hydrolase. Journal of Biochemical and Biophysical Methods 67: 131140.

Mandrich L, Manco G, Rossi M, Floris E, Bosch T, Smit $G$ \& Wouters J. 2006. Alicyclobacillus acidocaldarius Thermophilic Esterase EST2's Activity in Milk and Cheese Models. Applied and Environmental Microbiology 72: 3191-3197.

Mandrich L, Merone L, Pezzullo M, Cipolla L, Nicotra F, Rossi M \& Manco G. 2005. Role of the $\mathrm{N}$ terminus in enzyme activity, Stability and Specificity in thermophilic esterases belonging to the HSL family. Journal of Molecular Biology 345: 501-512.

Martínez-Martínez I, Navarro-Fernández J, LozadaRamírez JD, García-Carmona F \& Sánchez-Ferrer A. 2008. YesT: A new rhamnogalacturonan acetyl esterase from Bacillus subtilis. Proteins 71: 379-388.

Mnisi S \& Theron M. 2005. Cloning and characterization of a carboxylesterase from Bacillus coagulans 8111. Current Microbiology 50: 196-201.

Nawani N, Khurana J \& Kaur J. 2006. A thermostable lipolytic enzyme from a thermophilic Bacillus sp.: $\mathrm{Pu}-$ rification and characterization. Molecular and Cellular Biochemistry 290: 17-22.

Park Y, Choi SY \& Lee H.B. 2006. A carboxylesterase from the thermoacidophilic archaeon Sulfolobus solfataricus $\mathrm{P} 1$; purification, characterization, and expression. Biochimica et Biophysica Acta 1760: 820828.

Prim N, Blanco A, Martinez J, Pastor F \& Diaz P. 2000. EstA, a gene coding for a cell-bound esterase from
Paenibacillus sp. BP-23, is a new member of the bacterial subclass of type $B$ carboxylesterase. Research in Microbiology 151: 303-312.

Pühler A \& Tauch A. 2003. A new era in Corynebacterium glutamicum biotechnology. Journal of Biotechnology 104: 1-3.

Ruckert C, Puhler A \& Kalinowski J. 2003. Genome-wide analysis of the L-methionine biosynthetic pathway in Corynebacterium glutamicum by targeted gene deletion and homologous complementation. Journal of Biotechnology 104: 213-228.

Reetz MT. 2004. Controlling the enantioselectivity of enzyme by directed evolution: Practical and theoretical ramifications. Proceedings of the National Academy of Sciences 101: 5716-5722.

Sanchez-Ferrer A, Martinez-Martinez I, García-Pizarro E \& García-Carmona F. 2007. Producción de formas quiméricas de cefalosporina C acetilasa. ES2272115.

Sambrook J, Fritsch EF \& Maniatis T. 1989. Molecular cloning: a laboratory manual, 2nd ed. Cold Spring Harbor Laboratory Press, Cold Spring Harbor, NY.

Smith PK, Kroha RI, Hermanson GT, Mallia AK, Gartner FH, Provenzano MD, Fujimoto EK, Goeke NM, Olson BJ \& Klenk DC. 1985. Measurement of protein using bicinchoninic acid. Analytical Biochemistry 150: $76-85$.

Suzuki Y, Miyamoto K \& Ohta H. 2004. A novel thermostable esterase from the thermoacidophilic archaeon Sulfolobus tokodaii strain 7. FEMS Microbiology Letters 236: 97-102.

Undurraga D, Markovits A \& Erazo S. 2001. Cocoa butter equivalent throught enzymatic interesterification of palm oil midfraction. Process Biochemistry 36: 933-939. 